\title{
Use of pulmonary embolism rule-out criteria (PERC) in the emergency department
}

\author{
Authors: Richard Siau, Steven Young, Melissa Blyth and Kirsty Dickson-Jardine
}

\begin{abstract}
Aims
To assess the use of pulmonary embolism rule-out criteria (PERC) to exclude pulmonary embolism (PE) in the emergency department.

\section{Methods}

An extensive literature review was carried out to evaluate the evidence base for the use of PERC scoring in the exclusion of PE. A retrospective study of case notes of all emergency department patients receiving D-dimer assays or undergoing computed tomography pulmonary angiography (CTPA) from 1-30 April 2014 was performed. Patients receiving D-dimer tests for the diagnosis of deep-vein thrombosis were excluded. Rates of documentation and PERC status were recorded and patients who were inappropriately investigated were identified.
\end{abstract}

\section{Results}

Literature search revealed that use of PERC scoring to complement clinical suspicion is a useful tool for exclusion of PE. Out of $68 \mathrm{D}$-dimer tests requested, only two patients had the PERC score documented. 17 D-dimer tests and one CTPA were ordered on patients in whom PE could have been excluded by PERC scoring.

\section{Conclusions}

The PERC rule has been validated as a safe way of excluding PE when used in conjunction with clinical suspicion. Encouraging use of PERC scoring in the acute setting may be an inexpensive way of reducing unnecessary testing, resulting in reduced emergency department waiting times and expenditure, in addition to reducing unnecessary patient irradiation.

\section{Conflict of interest statement}

There are no conflicts of interest.

Authors: Emergency Department, Morriston Hospital, 